\title{
Flux amplification using stochastic superconducting quantum interference devices
}

\author{
R. Rouse, Siyuan Han, and J. E. Lukens ${ }^{\text {a) }}$ \\ Department of Physics, SUNY at Stony Brook, Stony Brook, New York 11794-3800
}

(Received 25 August 1994; accepted for publication 28 October 1994)

\begin{abstract}
The flux change $\delta \Phi$ through a bistable superconducting quantum interference device has been measured in the presence of thermally induced switching (with rate $\Gamma$ ) versus $\delta \Phi_{x}$, the change in the applied flux. For small $\delta \Phi_{x}, \delta \Phi$ is proportional to $\delta \Phi_{x}$ with a measured flux gain $g$, depending on the temperature, barrier height, and frequency $\Omega$, with a maximum of about 16. In agreement with theories of periodically driven stochastic bistable systems, $g(\Omega)$ is nearly frequency independent up to $\Gamma$ and is proportional to $\Omega^{-1}$ for $\Omega \gg \Gamma$. For larger amplitude signals, harmonic generation has been measured in the adiabatic limit $(\Omega \ll \Gamma)$ and found to be in good agreement with theory. Possible applications of this system for flux measurement are discussed. (C) 1995 American Institute of Physics.
\end{abstract}

The superconducting quantum interference device (SQUID), a superconducting loop interrupted by one or two Josephson junctions, has proven to be an extraordinarily sensitive magnetometer (see, e.g., Ref. 1 for a review). Essentially, the operation of these devices involves switching the loop between fluxoid states using either a rf flux (single junction rf SQUID) or the Josephson oscillations resulting from a dc voltage across the junctions (two junction dc SQUID). In general, the noise per unit bandwidth is reduced as this switching frequency is increased. Recent theories of periodically driven stochastic bistable systems ${ }^{2-4}$ show that, in principle, high sensitivity can also be achieved if the intrinsic thermal fluctuations of the device are the source of this switching. In this letter, we apply these results to a SQUID system and demonstrate that substantial flux gain can be achieved in both passive and active versions of the system.

The system being studied here, which we refer to as a stochastic (or S-) SQUID, uses the rf SQUID configuration, i.e., a superconducting loop of inductance $L$ interrupted by a Josephson junction of critical current $I_{c}{ }^{5}$ The equation of motion for the flux $\Phi$ through this S-SQUID is homologous to that of a particle of mass $C$ (junction's shunt capacitance), moving in a potential $U(\Phi)$ and having a friction coefficient $\gamma=1 / R$, where $R$ is the junction's shunt resistance. This potential, shown in Fig. 1 (insert), is given by:

$$
U(\Phi)=\frac{\left(\Phi-\Phi_{x}\right)^{2}}{2 L}+E_{J} \cos \left(2 \pi \frac{\Phi}{\Phi_{0}}\right)
$$

Here, $\Phi_{x}$ is the externally applied magnetic flux through the SQUID. $\Phi_{0}$ is the flux quantum, and $E_{J}=\Phi_{0} I_{c} / 2 \pi$ is the maximum Josephson coupling energy of the junction. In Eq. (1) the origins of $\Phi$ and $\Phi_{x}$ have been chosen at $\Phi_{0} / 2$ so that at $\Phi_{x}=0$ the potential is symmetric in $\Phi$ with two minima at $\pm \Phi_{m}$ separated by a barrier $\Delta U$ [cf. Fig. 1 (insert)]. For $\Phi_{x} \neq 0$, the symmetry of the potential is broken and an energy difference $2 \epsilon$ between the left and right wells appears with $\epsilon=\Phi_{m} \Phi_{x} / L$ for small $\Phi_{x}$.

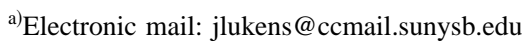

Thermally induced switching between the two wells occurs with a rate given (for $\Delta U / k_{B} T \gg 1$ ) by the Kramers relation as

$$
\Gamma=\Gamma_{0} \exp \left(\frac{-\Delta U}{k_{B} T}\right) .
$$

$\Gamma_{0}$ depends on the S-SQUID parameters with values approaching $10^{12} \mathrm{~s}^{-1}$ possible. This switching will produce random telegraph noise in $\Phi$ with a spectral density given by

$$
S_{\Phi}^{(N)}(\omega)=\frac{\Phi_{m}^{2} \tau}{2 \pi\left[1+(\omega \tau / 2)^{2}\right]},
$$

where $\tau=1 / \Gamma$. Theory ${ }^{2,4}$ shows that the addition of a small sinusoidal energy modulation of the form $\epsilon=\epsilon_{0} \sin (\Omega t)$ [i.e., $\left.\Phi_{x}=\tilde{\Phi}_{x} \sin (\Omega t)\right]$ to such a system results in a total spectral density $S_{\Phi}(\omega)$ given by Eq. (3) plus $\delta$ peaks at $\Omega$ and its harmonics. The spectral weights $S_{\Phi}^{(S)}(\Omega)$ of these $\delta$ peaks have been calculated for a two level system with the result for the fundamental that

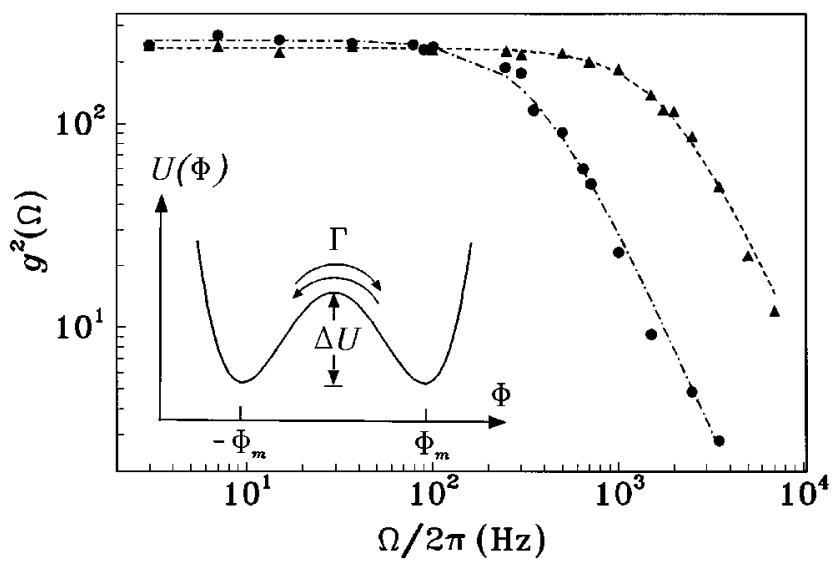

FIG. 1. Spectral weight of the first harmonic of the S-SQUID response to a sinusoidal applied flux at frequency $\Omega$. The two curves are for different barrier heights (circles-higher barrier, triangles-lower barrier) with resulting differences in $\tau$. The lines are the fit of the data to Eq. (4) using the values of $\tau$ obtained from the corresponding noise spectra. The insert shows the S-SQUID potential for $\Phi_{x}=0$. 


$$
S_{\Phi}^{(S)}(\Omega)=\frac{\left(\Phi_{m} \beta \epsilon_{0}\right)^{2}}{1+(\Omega \tau / 2)^{2}},
$$

where $\beta \equiv 1 / k_{B} T$. Thus, the signal response is also Lorentzian with the same cutoff frequency, determined by $\tau$, as the noise. As can be seen from Eq. (4), at low frequencies the signal gain, $\sqrt{S_{\Phi}^{(S)}(\Omega) / \tilde{\Phi}_{x}^{2}}$, is given by

$$
g(0)=\frac{\Phi_{m}^{2}}{L k_{B} T}
$$

Again, this is valid only in the small signal limit where $\Phi \ll \Phi_{m}$.

Measurements were made in a ${ }^{4} \mathrm{He}$ cryostat, using a setup similar to that described in detail elsewhere. ${ }^{5,6}$ In the S-SQUID $(L=200 p \mathrm{H})$, the single junction is replaced by two junctions in parallel in a low inductance $\left(L^{\prime}=10 \mathrm{pH}\right)$ superconducting loop. ${ }^{7,8}$ This sample is well described by Eq. (1) with a coupling energy $E_{J}$, which can be varied in situ by changing the flux through the small loop. This permits $\Delta U$ and thus $\Gamma$ to be varied at fixed temperature. In order to ensure that intrinsic fluctuations are the source of the transition, the sample is carefully shielded and is weakly coupled to the field coils used to modulate $\Phi_{x}$ and $E_{J}$ and to a dc SQUID magnetometer used to measure $\Phi$. Figure 1 shows the measured square of the small signal gain $g$ versus $\Omega$ for two barrier heights at $T=3.0 \mathrm{~K}$. The lines show the fit to Eq. (4) using the values of $\tau$ obtained by fitting the corresponding noise spectra to Eq. (3). As can be seen, the agreement is excellent giving values of $\Phi_{m}=0.178 \quad \Phi_{0}$ and $\Phi_{m}=0.183 \Phi_{0}$ for the low and high barrier cases, respectively.

It is clear from Eq. (4) that for a given signal frequency $\Omega$ the maximum gain is achieved for $\Gamma \gg \Omega$. Increasing $\Gamma$ by reducing $\Delta U$ or increasing $T$ also reduces the noise $S_{\Phi}^{(N)}(\Omega)$ thus increasing the signal-to-noise ratio, at least as long as Eq. (2) is valid. In this so-called adiabatic limit $(\Gamma \gg \Omega)$, the analysis of the system is straightforward even for larger signal amplitudes. Here, one can assume that thermal equilibrium between the wells is maintained at all times during the variation of $\Phi_{x}$ giving

$$
\langle\Phi(t)\rangle=\Phi_{m} \tanh \left(\frac{\Phi_{m} \Phi_{x}(t)}{L k_{B} T}\right) .
$$

While Eq. (6) is rigorously true only for a discrete two level system, it is an excellent approximation for the S-SQUID studied here as can be seen by the agreement of the measured static $\Phi-\Phi_{x}$ curve with Eq. (6) shown in Fig. 2. The gain, $d \Phi / d \Phi_{x}$, obtained from Eq. (6) also agrees with that given in Eq. (5). As an example, a SQUID with $L=64 p \mathrm{H}$ and $I_{c}=6.9 \mu \mathrm{A}$ would have a small signal gain of 48 at $4.2 \mathrm{~K}$ with a sensitivity of $0.1 \mu \Phi_{0} / \sqrt{\mathrm{Hz}}$, assuming the noise is given by Eq. (3). Of course, another device such as a dc SQUID would be required for reading out the flux so amplified. A detailed analysis shows that if the S-SQUID is coupled to the dc SQUID through a superconducting transformer, the dc SQUID noise is not amplified by the S-SQUID. Thus the signal-to-noise ratio of the system can easily be that due to the S-SQUID itself. This avoids the problems of shunt resistors and heating associated with dc

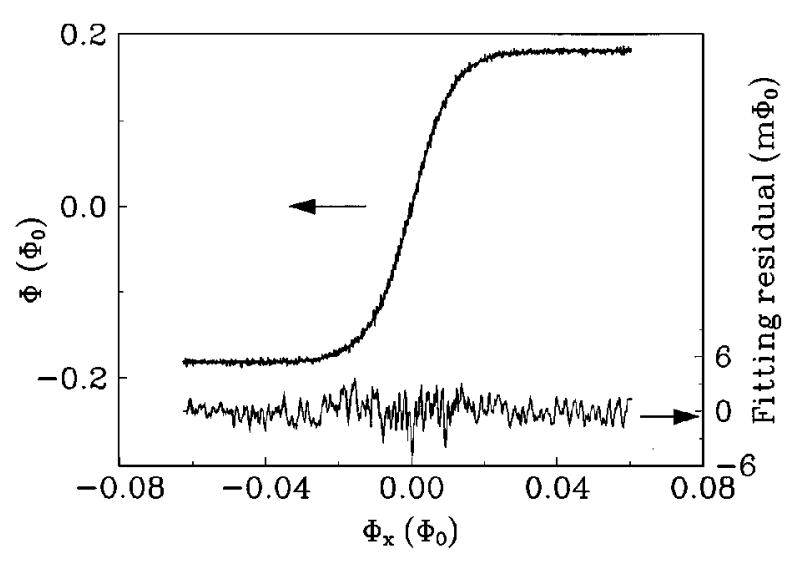

FIG. 2. Measured best fit of $\Phi$ vs $\Phi_{x}$ for large $\Gamma$ to Eq. (6) along with the resulting residual.

SQUIDs. The signal gain provided by the S-SQUID also considerably relaxes the stringent requirements for low noise amplifiers usually associated with dc SQUID systems.

Another mode of operation for the S-SQUID is based on the selection rules of periodically driven stochastic bistable systems first introduced by Jung and Hänggi. ${ }^{3}$ These rules state that when an external additive periodic driving force [in our case $\left.\tilde{\Phi}_{x} \sin (\Omega t)\right]$ of arbitrary amplitude is applied to a stochastic bistable system of even parity (e.g., the S-SQUID with dc flux $\bar{\Phi}_{x}=0$ ) the power spectral density of the system contains $\delta$ peaks only at $\Omega$ and its odd harmonics. For a bistable system having no definitive parity the power spectral density in general contains peaks at both odd and even harmonics. This effect can be clearly seen in the spectra shown in Figs. 3(a) and 3(b), where a flux modulation of amplitude $\tilde{\Phi}_{x}=42.5 \mathrm{~m} \Phi_{0}$ and frequency $\Omega / 2 \pi=53 \mathrm{~Hz}$ was applied to the S-SQUID. The spectrum in Fig. 3(a), which shows no

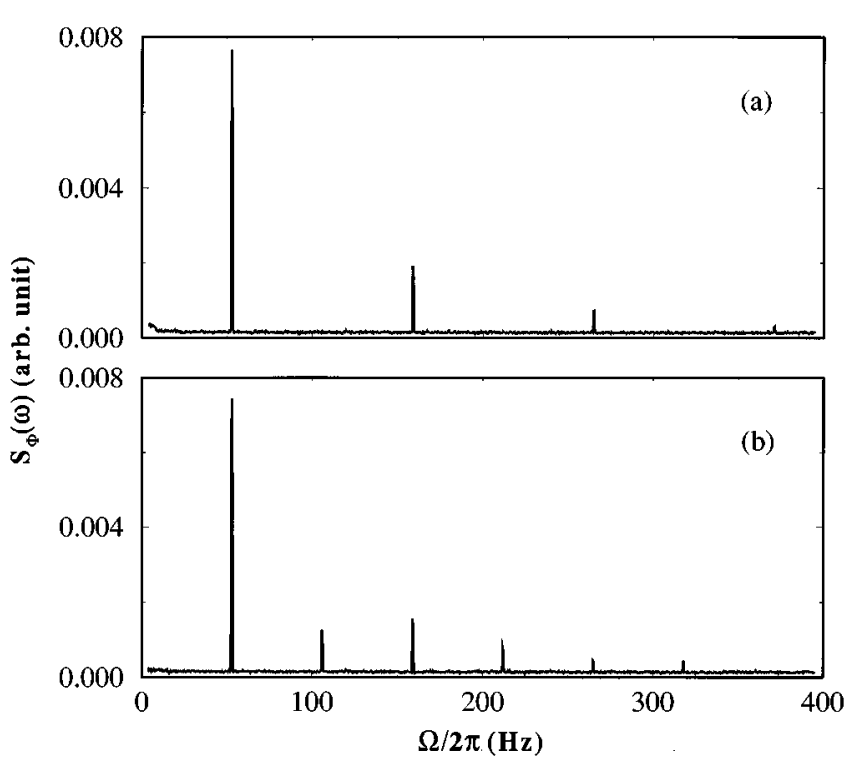

FIG. 3. Spectra of $\Phi$ resulting from large amplitude modulation of the S-SQUID potential by $\tilde{\Phi}_{x}$ for (a) $\bar{\Phi}_{x}=0$ and (b) $\bar{\Phi}_{x}=8.5 \mathrm{~m} \Phi_{0}$. Note the absence of even harmonics for the symmetric potential with $\bar{\Phi}_{x}=0$. 


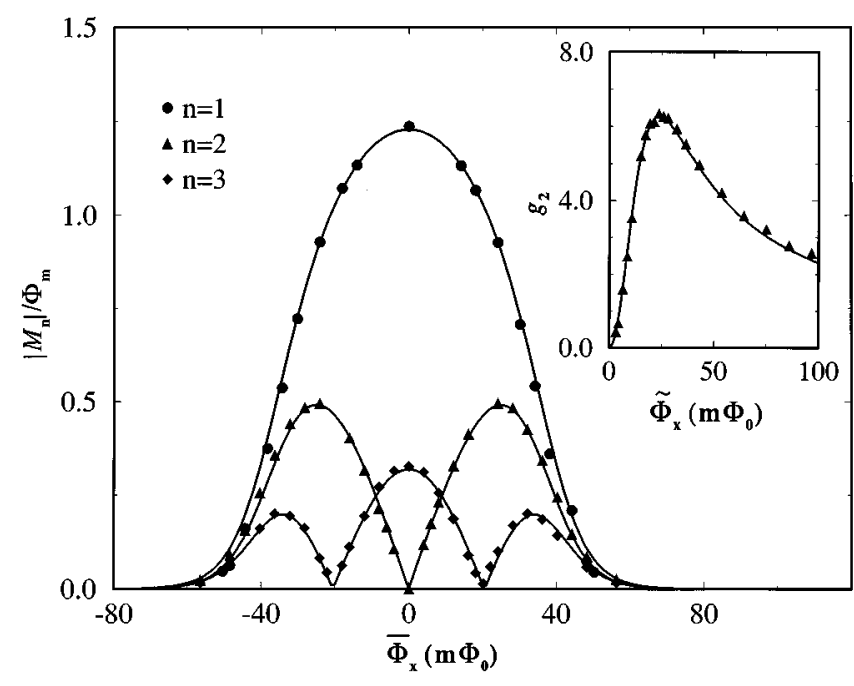

FIG. 4. The absolute value of the amplitude of the first three Fourier components (normalized to $\Phi_{m}$ ) of the S-SQUID response to a large amplitude signal $\tilde{\Phi}_{x}$ as a function of the mean applied flux $\bar{\Phi}_{x}$. The solid lines are the predictions of Eq. (7) with no adjustable parameters. The insert shows the slope of the second harmonic near $\bar{\Phi}_{x}=0$ as a function of the modulation amplitude $\tilde{\Phi}_{x}$ compared to that calculated from Eq. (7) again, with no adjustable parameters.

even harmonics, was obtained with $\bar{\Phi}_{x}=0$, while $\bar{\Phi}_{x}=8.5$ $\mathrm{m} \Phi_{0}$ for Fig. 3(b), which contains all harmonics.

In the adiabatic limit it is straightforward to calculate the amplitude of the harmonics. We obtain for the $n$th Fourier coefficient of the response, $M_{n}$

$$
M_{n}=\frac{\Omega}{\pi} \int_{-\pi / \Omega}^{\pi / \Omega} \Phi_{m} \tanh \left(\frac{\Phi_{m} \Phi_{x}(t)}{L k_{B} T}\right) \exp (i n \Omega t) d t
$$

where $\Phi_{x}(t)=\bar{\Phi}_{x}+\tilde{\Phi}_{x} \sin (\Omega t) . S_{\Phi}^{(S)}(n \Omega)=\left|M_{n}(\Omega)\right|^{2}$. Figure 4 shows the measured harmonic response of the S-SQUID as a function of $\bar{\Phi}_{x}$ for the first three harmonics, ${ }^{9}$ compared to that predicted from Eq. (7), with no adjustable parameters. Care was taken to ensure that all power from a given peak was registered in a single bin of the spectrum analyzer. The normalized spectral weights of the peaks plotted in Fig. 4 are calculated from the recorded spectral density times the bin size in hertz.

Since $M_{2}$ is a linear function of $\bar{\Phi}_{x}$ for small $\bar{\Phi}_{x}$, the amplitude of the second harmonic can serve as a measure of the low frequency $(\ll \Omega)$ flux applied to the S-SQUID. The phase of this signal shifts by $\pi$ as $\bar{\Phi}_{x}$ changes sign, and so can serve as the basis for a feedback scheme using phase sensitive detection for the measurement of large signals. One can define a second gain parameter for the system: $g_{2}\left(\tilde{\Phi}_{x}\right)=d M_{2}\left(\bar{\Phi}_{x}\right) / d \bar{\Phi}_{x}$. Figure 4 (insert) shows the measured $g_{2}\left(\tilde{\Phi}_{x}\right)$ for $\bar{\Phi}_{x}=4.32 \mathrm{~m} \Phi_{0}$ and $\Omega / 2 \pi=250 \mathrm{~Hz} \ll \Gamma$. The solid line is the prediction from Eq. (7) with all parameters independently measured. As can be seen, the maximum value of $g_{2}$, obtained by optimizing $\tilde{\Phi}_{x}$ is of the same order as $g$, so the sensitivity calculated above for the fundamental mode of operation would serve as a guide to the second harmonic mode as well.

In summary, we have demonstrated a flux gain of about 16 in a stochastic SQUID (using thermally activated flux switching). Theories of the signal to noise ratio in stochastic bistable systems, applied to this S-SQUID, indicate that flux gains much greater than 100 should be possible with a sensitivity greater than $10^{-7} \Phi_{0} / \sqrt{\mathrm{Hz}}$. The large signal response of the S-SQUID has been analyzed and measured in the adiabatic limit with excellent agreement obtained. The substantial second harmonic gain shows that the S-SQUID can be used in a flux-locked loop system.

The authors are grateful for helpful discussions with Peter Hänggi and thank Sue Coppersmith for bringing this subject to our attention. ${ }^{10}$ This work is supported by the U.S. Office of Naval Research.

${ }^{1} \mathrm{~J}$. Clarke, in The New Superconducting Electronics, edited by $\mathrm{H}$. Weinstock and R. W. Ralston (Kluwer, Boston, 1993), Chap. 5, pp. $123-$ 180.

${ }^{2}$ B. McNamara and K. Wiesenfeld, Phys. Rev. A 39, 4854 (1989).

${ }^{3}$ P. Jung and P. Hänggi, Phys. Rev. A 44, 8032 (1991).

${ }^{4}$ P. Jung, Phys. Rep. 234, 175 (1993), and references therein.

${ }^{5}$ S. Han, J. Lapointe, and J. Lukens, in Activated Barrier Crossing, 1st ed., edited by G. Fleming and P. Hänggi (World Scientific, Singapore, 1993), Chap. 9, pp. 241-267.

${ }^{6}$ J. Lapointe, Ph.D. dissertation, State University of New York at Stony Brook, Department of Physics, 1993.

${ }^{7}$ S. Han, J. Lapointe, and J. E. Lukens, Phys. Rev. Lett. 63, 1712 (1989).

${ }^{8}$ B. K. Sen, Ph.D. dissertation, State University of New York at Stony Brook, Department of Physics, 1986.

${ }^{9}$ The null in the $n=3$ response (Fig. 4) has recently been predicted as part of a more detailed analysis: R. Bartussek, P. Jung, and P. Hänggi, Phys. Rev. E 49, 3930 (1994).

${ }^{10}$ Note added in proof. We thank A. R. Bulsara for sending us, prior to publication, his paper on stochastic resonance in SQUIDs: A. D. Hibbs, A. L. Singsaas, E. W. Jacobs, A. R. Bulsara, J. J. Bekkedahl, and F. Moss, J. Appl. Phys. (in press). 